\title{
USO DE METODOLOGIAS ATIVAS DE APRENDIZAGEM PARA A EDUCAÇÃO NA SAÚDE: ANÁLISE DA PRODUÇÃO CIENTÍFICA
}

\author{
USING ACTIVE LEARNING METHODOLOGIES FOR EDUCATION IN HEALTH:SCIENTIFIC PRODUCTION \\ ANALYSIS
}

\author{
USO DE METODOLOGÍAS ACTIVAS DE APRENDIZAJE PARA LA EDUCACIÓN EN LA SALUD: \\ ANÁLISIS DE LA PRODUCCIÓN CIENTÍFICA
}

\author{
Cilene Maria Freitas ${ }^{1}$ \\ Cibelly Aliny Siqueira Lima Freitas ${ }^{2}$ \\ José Reginaldo Feijão Parente ${ }^{3}$ \\ Maristela Inês Osawa Vasconcelos ${ }^{4}$ \\ Gleiciane Kélen Lima ${ }^{5}$ \\ Karina Oliveira de Mesquita ${ }^{6}$ \\ Svetlana Coelho Martins ${ }^{7}$ \\ Janice Dávila Rodrigues Mendes ${ }^{8}$
}

Resumo o presente artigo tem como objetivo principal analisar a produção científica sobre o uso de metodologias ativas de aprendizagem na educação na saúde. Trata-se de uma revisão bibliográfica da literatura utilizando-se para seleção dos artigos a base de dados da Biblioteca Virtual em Saúde. A pesquisa ocorreu nos meses de setembro a novembro de 2012. Na busca selecionaram-se 15 artigos completos. Os resultados evidenciaram que o uso das metodologias ativas motiva o discente e o direciona para buscar informações no intuito de solucionar impasse e promover seu próprio desenvolvimento, fazendo com que ele perceba que a nova aprendizagem é um instrumento necessário e significativo para ampliar suas possibilidades e caminhos. Essas metodologias também têm contribuído para mudanças no modo de pensar e agir dos profissionais de saúde, os quais passaram a interagir em um espaço plural de interesses, potencialidades e capacidades.

Palavras-chave metodologias ativas de aprendizagem; educação na saúde.
Abstract This article aims to review the scientific literature on the use of active learning methodologies in health education. This is a review of the literature using the Virtual Health Library database to select the articles. The survey took place from September to November 2012. Fifteen full articles were selected in the search. The results showed that using active methodologies motivates students and directs them to seek information to solve impasses and further their own development, making them realize that new learning is a necessary, significant tool to enhance their possibilities and paths. These methodologies have also contributed to changes in the health professionals' way of thinking and acting, who started to interact in a plural space of interests, potentials, and capabilities. Keywords active learning methodologies; health education. 


\section{Introdução}

O ensino na saúde, nas últimas décadas, vem passando por um processo de ampla rediscussão, com o objetivo de formar um profissional com mais possibilidades de agir na resolução dos problemas de saúde dos indivíduos e da coletividade (Brasil, 2001). Os modelos de ensino tradicionais levam o aluno a uma postura quase sempre passiva, ou seja, sem a oportunidade de demonstrar suas opiniões, interesses e de repassar seus saberes também para o docente, através de uma comunicação mútua.

Assim, para integrar teoria e prática, serviço e ensino, as propostas metodológicas usadas na formação dos profissionais da saúde devem ser repensadas, tornando-se premente uma abordagem ampliada e integrada dos currículos, priorizando a formação de competências e o estímulo à utilização de metodologias ativas de aprendizagem, criando profissionais com ampla visão do homem (Mitre et al., 2008).

As metodologias ativas se caracterizam por colocar o estudante no centro do processo de ensino-aprendizagem, tornando-o construtor do seu próprio conhecimento por meio de um currículo que agrega as diferentes disciplinas, permitindo que ele desenvolva um olhar amplo acerca do ser humano, nas suas relações com a sociedade e com o ambiente (Siqueira-Batista, Rodrigo e Siqueira-Batista, Rômulo, 2009). Para a inovação do currículo é necessária a elaboração de estratégias que visem à articulação entre a teoria e a prática no momento do processo de ensino, para que os estudantes possam criar concepções e construir seu próprio modelo de aprendizagem.

Segundo Munguba (2010), ao aplicar estratégias inovadoras, a postura de quem ensina deve ser revisitada sistematicamente, visando evitar incoerências, perceptíveis às pessoas alvo da ação. A práxis ao desenvolver a dinâmica de aprender e ensinar requer a oportunidade e o estímulo ao exercício da liberdade de expressão, de ação e o diálogo para todos os envolvidos.

Para Haguenauer (2005), os métodos de ensino ultrapassados podem empobrecer a criatividade e a inteligência dos jovens. Ao mesmo tempo, a autora afirma que a eficiência da aprendizagem nas universidades e na capacitação de profissionais é muito baixa quando são utilizados os métodos tradicionais. Logo, é preciso modernizar a educação para acompanhar as transformações ocorridas no mundo. Considerando a importância da aplicação de metodologias que contribuam e inovem o processo de ensino-aprendizagem no âmbito acadêmico e diante da necessidade de promover uma discussão com ênfase nessas ferramentas e no seu impacto para a educação na saúde, justifica-se a realização de uma pesquisa bibliográfica.

Atendendo a prerrogativa das políticas de educação na saúde, como a ordenação da formação de recursos humanos para o Sistema Único de Saúde (SUS), espera-se que este trabalho possa contribuir para uma abordagem 
integrada e ampliada dos currículos na formação desses profissionais, priorizando a utilização de estratégias metodológicas inovadoras que permitam o desenvolvimento de um egresso com perfil capaz de mobilizar mudanças no e para o processo de trabalho, no sentido de dar resolutividade aos problemas de saúde da população. Desse modo, o presente artigo teve por objetivo analisar a produção científica sobre o uso de metodologias ativas de aprendizagem na educação na saúde.

\section{Procedimentos metodológicos}

Para condução desta investigação, adotou-se a revisão bibliográfica da literatura, objetivando colocar o pesquisador em contato direto com aquilo que foi escrito sobre determinado assunto (Marconi e Lakatos, 2004). Nesse sentido, esta revisão seguiu as seguintes etapas: seleção da pergunta norteadora; estabelecimento de critérios de inclusão e exclusão de artigos (seleção da amostra); definição das informações a serem extraídas dos artigos selecionados; análise dos resultados; e discussão e apresentação dos resultados.

Formulou-se a seguinte questão para guiar a revisão bibliográfica: De que forma as metodologias ativas de aprendizagem contribuem e se configuram em potenciais estratégias para a educação na saúde?

O levantamento das produções bibliográficas ocorreu nos meses de setembro a novembro de 2012, sendo utilizadas para pesquisa as bases de dados da Biblioteca Virtual em Saúde (BVS).

Na ocasião, foram empregados os seguintes critérios de inclusão dos artigos definidos: artigos disponíveis eletronicamente publicados entre os anos de 2001 a 2012; artigos disponíveis no idioma português; e artigos completos que abordam os fundamentos teóricos das metodologias ativas de aprendizagem e educação na saúde. Foram excluídos durante a busca: toda produção duplicada, editoriais, cartas ao editor, boletins epidemiológicos, bem como estudos que não abordem temática relevante ao alcance do objetivo da revisão.

Os termos utilizados para a busca dos artigos na BVS foram: metodologias ativas de aprendizagem e educação na saúde. Inicialmente, eles foram consultados nos Descritores em Ciências da Saúde (DeCS) e, embora não encontrados, permanecemos com esses termos para a busca de artigos, uma vez que atendiam ao objetivo desta revisão.

A busca foi realizada pelo acesso online, utilizando um instrumento que contempla os seguintes itens: identificação do artigo original, características metodológicas do estudo, avaliação do rigor metodológico e dos resultados encontrados, dentre outras informações. Os artigos foram lidos na íntegra e analisados quanto aos aspectos quantitativos e qualitativos. Em termos quantitativos, os dados foram analisados descritivamente em números absolutos, 
dispostos em quadros, compostos das seguintes variáveis: periódicos; artigos; autores; ano de publicação; país/estado; e tipo do estudo.

Em termos qualitativos, os indicadores foram recolhidos por meio da leitura exploratória, seletiva, analítica e interpretativa, tendo como premissas as discussões que envolvem metodologias inovadoras do ensino na saúde.

\section{Resultados e discussão}

A análise da produção bibliográfica sobre as metodologias ativas de aprendizagem na educação na saúde ocorreu a partir das bases de dados da BVS. Nelas foram encontrados 15 artigos completos com o cruzamento dos termos: metodologias ativas de aprendizagem e educação na saúde, como apresentados na Tabela 1.

Tabela 1

Produção científica sobre metodologias ativas de aprendizagem e educação na saúde

\begin{tabular}{|c|c|c|c|c|c|}
\hline Periódico & Artigo & Autores & Ano & País/Estado & Tipo de estudo \\
\hline $\begin{array}{l}\text { Revista Brasileira de } \\
\text { Educação Médica }\end{array}$ & $\begin{array}{l}\text { Avaliação do desenvolvimento de atitudes } \\
\text { humanísticas na graduação médica }\end{array}$ & Silvia Caixeta de Andrade et al. & 2011 & $\begin{array}{l}\text { Brasil/Rio } \\
\text { de Janeiro }\end{array}$ & Quantitativo \\
\hline $\begin{array}{l}\text { Interface: Comunicação, } \\
\text { Saúde e Educação }\end{array}$ & $\begin{array}{l}\text { Pós-graduação multiprofissional em saúde: resultados } \\
\text { de experiências utilizando metodologias ativas }\end{array}$ & Maria José Sanches Marin et al. & 2010 & $\begin{array}{l}\text { Brasil/São } \\
\text { Paulo }\end{array}$ & $\begin{array}{l}\text { Quantitativo e } \\
\text { Qualitativo }\end{array}$ \\
\hline Ciência \& Educação & $\begin{array}{l}\text { O uso de metodologias ativas no ensino de graduação } \\
\text { nas ciências sociais e da saúde - avaliação dos estudantes }\end{array}$ & Maria Paula Cerqueira Gomes et al. & 2010 & $\begin{array}{l}\text { Brasil/Rio } \\
\text { de Janeiro }\end{array}$ & Quantitativo \\
\hline $\begin{array}{l}\text { Revista Brasileira de } \\
\text { Educação Médica }\end{array}$ & $\begin{array}{l}\text { O ensino da ética nos cursos de graduação da área } \\
\text { de saúde }\end{array}$ & Larissa Arbués Carneiro et al. & 2010 & $\begin{array}{l}\text { Brasil/ } \\
\text { Brasília }\end{array}$ & Quantitativo \\
\hline $\begin{array}{l}\text { Revista Brasileira de } \\
\text { Educação Médica }\end{array}$ & $\begin{array}{l}\text { Transição paradigmática na educação médica: um olhar } \\
\text { construtivista dirigido à aprendizagem baseada em problemas }\end{array}$ & Pedro Henrique Netto Cezar et al. & 2010 & $\begin{array}{l}\text { Brasil/Rio } \\
\text { de Janeiro }\end{array}$ & $\begin{array}{l}\text { Revisão } \\
\text { bibliográfica }\end{array}$ \\
\hline $\begin{array}{l}\text { Trabalho, Educação e } \\
\text { Saúde }\end{array}$ & $\begin{array}{l}\text { Uso de metodologias ativas na formação técnica do } \\
\text { agente comunitário de saúde }\end{array}$ & Ivanilda Lacerda Pedrosa et al. & 2011 & $\begin{array}{l}\text { Brasil/Rio } \\
\text { de Janeiro }\end{array}$ & Relato \\
\hline Saúde e Sociedade & $\begin{array}{l}\text { Formação dos profissionais de saúde para o SUS: } \\
\text { significado e cuidado }\end{array}$ & $\begin{array}{l}\text { Karina Barros Calife Batista; Otília } \\
\text { Simões Janeiro Gonçalves. }\end{array}$ & 2011 & $\begin{array}{l}\text { Brasil/São } \\
\text { Paulo }\end{array}$ & $\begin{array}{l}\text { Revisão de } \\
\text { literatura }\end{array}$ \\
\hline Aquichan & $\begin{array}{l}\text { Educação permanente e qualidade da assistência à } \\
\text { saúde: aprendizagem significativa no trabalho da } \\
\text { enfermagem }\end{array}$ & $\begin{array}{l}\text { Fernanda Maria do Carmo da } \\
\text { Silveira Neves de Oliveira }\end{array}$ & 2011 & $\begin{array}{l}\text { Colômbia/ } \\
\text { Chía }\end{array}$ & $\begin{array}{l}\text { Revisão } \\
\text { integrativa }\end{array}$ \\
\hline
\end{tabular}


Continuação - Tabela 1

Produção científica sobre metodologias ativas de aprendizagem e educação na saúde

\begin{tabular}{|c|c|c|c|c|c|}
\hline Periódico & Artigo & Autores & Ano & País/Estado & Tipo de estudo \\
\hline $\begin{array}{l}\text { Revista Brasileira em } \\
\text { Promoção da Saúde }\end{array}$ & $\begin{array}{l}\text { Educação na saúde - sobreposição de saberes ou } \\
\text { interface? }\end{array}$ & $\begin{array}{l}\text { Marilene Calderaro da Silva } \\
\text { Munguba }\end{array}$ & 2010 & Brasil/Ceará & Revisão \\
\hline $\begin{array}{l}\text { Revista da Faculdade } \\
\text { de Odontologia }\end{array}$ & $\begin{array}{l}\text { Mudança no processo ensino-aprendizagem nos } \\
\text { cursos de graduação em odontologia com utilização } \\
\text { de metodologias ativas de ensino e aprendizagem }\end{array}$ & Valéria da Penha Freitas et al. & 2009 & $\begin{array}{l}\text { Brasil/Rio } \\
\text { Grande do } \\
\quad \text { Sul }\end{array}$ & $\begin{array}{l}\text { Revisão de } \\
\text { literatura }\end{array}$ \\
\hline $\begin{array}{l}\text { Revista da Associação } \\
\text { Médica de Minas Gerais }\end{array}$ & $\begin{array}{l}\text { Novas metodologias de integração do ensino de } \\
\text { saúde pública na faculdade de medicina utilizando } \\
\text { a informática }\end{array}$ & Thiago Fuscaldi-Corrêa et al. & 2008 & $\begin{array}{l}\text { Brasil/Minas } \\
\text { Gerais }\end{array}$ & Quantitativo \\
\hline Ciência \& Saúde Coletiva & $\begin{array}{l}\text { Metodologias ativas de ensino-aprendizagem na } \\
\text { formação profissional em saúde: debates atuais }\end{array}$ & Sandra Minardi Mitre et al. & 2008 & - & Qualitativo \\
\hline $\begin{array}{l}\text { Comunicação, Ciências } \\
\text { Saúde }\end{array}$ & $\begin{array}{l}\text { Educação nutricional no Brasil: evolução e descrição } \\
\text { de proposta metodológica para escolas }\end{array}$ & $\begin{array}{l}\text { Lívia Penna Firme Rodrigues; } \\
\text { Maria José Roncada }\end{array}$ & 2008 & $\begin{array}{l}\text { Brasil/ } \\
\text { Brasília }\end{array}$ & $\begin{array}{c}\text { Revisão } \\
\text { bibliográfica }\end{array}$ \\
\hline Cogitare Enfermagem & $\begin{array}{l}\text { A formação de profissionais da saúde: aprendizagem } \\
\text { significativa à luz da promoção da saúde }\end{array}$ & Anna Maria Chiesa et al. & 2007 & $\begin{array}{l}\text { Brasil/São } \\
\text { Paulo }\end{array}$ & Revisão \\
\hline $\begin{array}{l}\text { Interface: Comunicação, } \\
\text { Saúde e Educação }\end{array}$ & $\begin{array}{l}\text { Contribuição ao movimento de mudança na formação } \\
\text { profissional em saúde: uma avaliação das experiências UNI }\end{array}$ & Laura Feuerwerker; Roseni Sena & 2002 & $\begin{array}{l}\text { Brasil/Minas } \\
\text { Gerais }\end{array}$ & Qualitativo \\
\hline
\end{tabular}

Fonte: Os autores.

Nota: UNI (Uma Nova Iniciativa na Educação dos Profissionais de Saúde: União com a Comunidade).

Os artigos selecionados foram examinados criteriosamente, culminando na formação de categorias temáticas, que se basearam no principal foco de cada estudo. Emergiram, assim, três categorias: Inserção de abordagens pedagógicas inovadoras nos currículos de graduação e na formação técnica; Aplicação de metodologias ativas na capacitação de profissionais da saúde; Contribuições das metodologias ativas de aprendizagem para a formação e/ou capacitação das profissões em saúde.

\section{Inserção de abordagens pedagógicas inovadoras nos currículos de graduação e na formação técnica}

A formação tradicional em saúde, ainda hoje utilizada, decorre das recomendações elaboradas por Flexner, em 1910, que foram amplamente difundidas e resultaram em uma espécie de 'modelo' a ser assumido pelas instituições 
formadoras. Desde então, a formação em saúde adota um ensino organizado em disciplinas, centrado no professor, com atividades práticas em cenário eminentemente hospitalar, marcado pela unidirecionalidade na relação professor-estudante e pela fragmentação do corpo e da saúde das pessoas. Esse 'modelo' é responsável pela formação de profissionais que dominam os mais variados tipos de tecnologias, mas que são pouco hábeis para lidar com as dimensões subjetivas, sociais e culturais das pessoas, e mostra-se cada vez mais distanciado do atual modelo de organização dos serviços da rede pública de saúde em nosso país (Ribeiro, 2005).

Muitos autores discorrem sobre a utilização das metodologias ativas no ensino de diversas disciplinas e áreas do conhecimento e, além disso, apresentam diversos tipos de metodologias que podem ser empregadas a fim de ajustar o perfil dos profissionais de saúde, onde os conhecimentos sejam construídos com base na prática concreta do trabalho em saúde e na reflexão crítica sobre essa prática.

Buscando atender a uma necessidade nacional, algumas instituições formadoras de profissionais de saúde vêm implementando cursos nessa modalidade e propondo mudanças curriculares e dos métodos de ensino/aprendizagem (Marin et al., 2010). É o caso dos cursos de graduação em medicina, que desde 1969 vêm empregando as metodologias ativas, consubstanciadas na Aprendizagem Baseada em Problemas (ABP), tendo sido os cursos pioneiros implantados na Universidade de McMaster, no Canadá, e na Universidade de Maastricht, na Holanda. Posteriormente, essas metodologias foram difundidas em países da Ásia, África e América Latina (Berbel, 1998). No Brasil, algumas escolas médicas vêm adotando a ABP, sendo a Faculdade de Medicina de Marília e a Universidade Estadual de Londrina duas pioneiras no processo. No estado do Rio de Janeiro, o Curso de Graduação em Medicina da Fundação Educacional Serra dos Órgãos - atual Centro Universitário Serra dos Órgãos - foi pioneiro na adoção da dinâmica da ABP, em 2005 (Siqueira-Batista, Rodrigo e Siqueira-Batista, Rômulo, 2009).

Mitre et al. (2008) afirmam que se torna premente uma abordagem ampliada e integrada dos currículos, priorizando a formação de competências e o estímulo à utilização de metodologias ativas de aprendizagem, criando profissionais com ampla visão do homem.

Andrade et al. (2011) discutem a inserção precoce dos estudantes no serviço que funciona como uma metodologia pedagógica que vem sendo seguida por diversas escolas médicas com a diversificação dos cenários de ensino-aprendizagem. Na Escola Superior de Ciências da Saúde, o estudante tem a possibilidade de atuar de forma incisiva na realidade de uma Unidade Básica de Saúde (UBS) durante três anos, por meio do Eixo Educacional Interação Ensino, Serviços e Comunidade. Inicialmente participa de visitas domiciliares junto a agentes comunitários de saúde (ACSs), posteriormente é 
envolvido nas diversas atividades realizadas na UBS e, finalmente, procura modificar e renovar modos de trabalho deficitários do cenário.

As mudanças nas estruturas curriculares nas instituições educacionais, utilizando as metodologias ativas por meio de Módulos Temáticos Interdisciplinares e Dinâmica Tutorial, Interação Ensino, Serviço e Comunidade e Estágio Curricular Obrigatório, permitem aos estudantes uma visão crítica sobre os mais variados assuntos aprendidos durante a graduação; além disso, possibilita a inserção do aluno no mercado de trabalho, o que favorece a aquisição de conhecimentos e experiências para a futura carreira profissional.

Cezar et al. (2010) citam como exemplos dessas metodologias a Problematização e a ABP. No entanto, as duas propostas se desenvolvem a partir de visões teóricas distintas e têm pontos convergentes e pontos divergentes. Berbel cita a Problematização como metodologia e a ABP como uma proposta de organização curricular. Afirma ainda que a Problematização, ao contrário das práticas educativas mais individuais ou individualizantes, é uma metodologia de ensino que concebe a educação como uma prática social (Berbel, 1998).

$\mathrm{O}$ autor segue discutindo sobre a metodologia da problematização, a qual sugere que os estudantes partam de uma realidade e retornem a ela de modo transformador. Aprende-se, portanto, que as ações que renovam uma comunidade devem ser pautadas em pesquisa científica, na observação de outras experiências e na formação de consciência crítica sobre o que é plausível ser aproveitado na realidade da comunidade assistida (Berbel, 1998).

A metodologia da problematização ainda é discorrida por outros autores (Siqueira, 2003; Figueira et al., 2004) que pesquisaram o ensino da ética nos cursos de graduação em saúde. Eles afirmam que algumas experiências e indicações em direção à integração da ética na graduação estão na utilização de metodologias ativas, como a aprendizagem baseada em problemas ou Problematização, como instrumentos de construção do conhecimento nos cursos de saúde.

Ressaltam que um currículo fragmentado contribui para uma formação igualmente fragmentada, o que traz consequências para o ensino da ética na graduação. Nesse sentido, a mudança curricular pode ser uma oportunidade para inserir a ética de maneira integrada nos mais variados níveis da formação dos estudantes de graduação (Athanazio et al., 2004). E, ainda, a reestruturação da universidade é apontada como um caminho para superar o modelo de formação especializado, que tem como algumas das consequências o descompromisso com os valores éticos, a fragmentação do conhecimento e o empobrecimento acadêmico (Siqueira, 2006 apud Carneiro et al., 2010).

Gomes, Moura e Amorim (2006) realizaram um estudo em que foram avaliadas duas instituições que passaram por estruturação ou reestruturação curricular recente e que tinham como um dos eixos a aprendizagem baseada 
em problemas (Problem Based Learning). Revelou-se que em apenas uma das instituições a ética aparece de forma transversal no currículo, e os autores atribuem esse dado ao modelo de ensino integrado adotado pela instituição.

As metodologias ativas vêm sendo empregadas na inovação dos currículos de diversos cursos, como nos cursos de graduação em medicina, odontologia, e na formação técnica de ACS. Pedrosa et al. (2011) discutem o uso de metodologias ativas na formação dos ACSs. Como recursos metodológicos para a apreensão dos conteúdos recorreu-se a estratégias pedagógicas inovadoras direcionadas para o perfil do educando/trabalhador. Essas estratégias levaram em consideração a utilização de métodos criativos, dinâmicos e reflexivos, postos em prática por meio dos seguintes procedimentos didáticos: problematização da realidade e do processo de trabalho, exercícios em grupo, plenárias, leitura comentada, exposições dialogadas, seminários, oficinas, debates temáticos, apresentação de filmes, interpretações musicais, dramatizações e estudos de casos.

Observa-se, assim, que a aplicação de metodologias ativas leva o discente a refletir sobre o seu processo de trabalho e a transformar a sua realidade, beneficiando-a, tendo em vista que desperta nele o senso crítico e a busca de mudanças em sua relação consigo mesmo, com o usuário e com a comunidade geral (Pedrosa et al., 2011). Dessa forma, o discente percebe que a nova aprendizagem é um instrumento necessário e significativo para ampliar suas possibilidades e caminhos.

Freitas et al. (2009) expõem no seu estudo as diferentes metodologias ativas que podem ser empregadas no processo de ensino e aprendizagem na Odontologia, que consistem no portfólio, a ABP e também meios de comunicação como a internet.

Nessa pesquisa, o ABP é mais uma vez enfatizado na literatura (Sakai e Lima, 1996) como sendo o eixo principal do aprendizado teórico do currículo de algumas escolas de graduação, em que o aprendizado é centrado no aluno.

\section{Aplicação de metodologias ativas na capacitação de profissionais de saúde}

O processo de formação baseado na metodologia tradicional impede que os profissionais de Saúde operem de modo a transformar as práticas de Saúde e organizar os serviços. Apresenta-se, desse modo, uma necessidade crescente de educação permanente para esses profissionais, com o objetivo de (re)significar seus perfis de atuação, para implantação e fortalecimento da atenção à Saúde no SUS, o que representa um grande desafio (Batista e Gonçalves, 2011).

A qualificação de Recursos Humanos na Saúde pode ser entendida como a efetivação de estratégias e ações para o aproveitamento do potencial dos 
profissionais, no sentido de enfrentar as mudanças e os desafios gerados no desempenho do trabalho diário nos diferentes espaços do SUS (Machado, 2003).

A Política Nacional de Educação Permanente em Saúde (PNEPS) enfoca a educação no serviço como o tipo de formação "mais apropriado para produzir as transformações nas práticas e nos contextos de trabalho, fortalecendo a reflexão na ação, o trabalho em equipes e a capacidade de gestão sobre os próprios processos locais" (Brasil, 2009).

O Ministério da Saúde tomou a Educação Permanente em Saúde como ideia central da Política de Gestão da Educação no trabalho em Saúde, passando a desenvolver ações indutoras significativas no interior do SUS, com a criação da Secretaria de Gestão do Trabalho e da Educação na Saúde no Ministério da Saúde. A proposta de Educação Permanente em Saúde que vem sendo implantada no Brasil destaca a importância do potencial educativo do processo de trabalho para a sua transformação. Busca a melhoria da qualidade do cuidado, a capacidade de comunicação e o compromisso social entre as equipes de saúde, os gestores do sistema de saúde, as instituições formadoras e o controle social. Estimula a produção de saberes a partir da valorização da experiência e da cultura do sujeito das práticas de trabalho em Saúde numa dada situação e com postura crítica (Ceccim, 2004).

A PNEPS aparece como uma proposta de ação estratégica para contribuir para a transformação e a qualificação das práticas de Saúde, a organização das ações e dos serviços, dos processos formativos e das práticas pedagógicas na formação e no desenvolvimento dos trabalhadores de Saúde. Implica trabalho intersetorial capaz de articular o desenvolvimento individual e institucional, as ações e os serviços, bem como a gestão local, a atenção à Saúde e o controle social (Brasil, 2004).

A partir de então, iniciaram-se diversas experiências no sentido de introduzir modelos de metodologias ativas na formação e qualificação dos trabalhadores de saúde. A parceria com o modelo atual possibilita que se somem as forças favoráveis à mudança existentes na academia, nos serviços e na comunidade, aumentando a governabilidade dos processos de mudança. Ao mesmo tempo, a própria interação produz um impulso em direção à mudança porque se evidenciam as limitações do modo tradicional de dar conta dos problemas da realidade agora percebidos sem tantos filtros.

Feuerwerker e Sena (2002), ao utilizarem Uma nova iniciativa na educação dos profissionais de saúde: união com a comunidade como metodologia ativa, pretendiam mudar os serviços de Saúde, contribuindo para que se tornassem mais efetivos, integrados, sensíveis à realidade local; mais abertos às necessidades da população e comprometidos com a resolução de problemas; com capacidade de participarem ativamente do processo de formação profissional.

Os autores pretenderam, também, contribuir para o fortalecimento da cidadania e da participação popular em saúde, ampliando o acesso da comunidade 
aos conhecimentos em saúde, às tecnologias fundamentais para seu desenvolvimento mais autônomo, fortalecendo suas organizações próprias e sua capacidade de intervenção local (Feuerwerker e Sena, 2002).

\section{Contribuiç̧̃es das metodologias ativas de aprendizagem para a formação e capacitação das profissões em saúde}

Encontramos em Paulo Freire (1996) uma defesa para as metodologias ativas, com sua afirmação de que, na educação de adultos, o que impulsiona a aprendizagem é a superação de desafios, a resolução de problemas e a construção do conhecimento novo a partir de conhecimentos e experiências prévias dos indivíduos. As metodologias ativas vão ao encontro da pedagogia da autonomia, que preconiza para a educação contemporânea um discente capaz de autogerenciar ou autogovernar o seu processo de formação (Freire, 2006).

Andrade et al. (2011) afirmam que alguns estudos atuais em educação médica têm demonstrado os benefícios associados a delineamentos curriculares mais inovadores, que adotam metodologias ativas de ensino, pequenos grupos de debate e inserção precoce dos estudantes nos serviços. Nesse novo modo de pensar e agir na formação dos profissionais de saúde, os atores do serviço e da comunidade passaram a interagir em um espaço plural de interesses, potencialidades e capacidades. Nesses novos espaços de interação definiram-se as novas responsabilidades conjuntas em relação ao processo ensino-aprendizagem e as específicas de cada instituição e de cada ator social. No campo das práticas pedagógicas as novas interações ampliaram os espaços de ação dos docentes e dos estudantes em realidades concretas (Feuerwerker e Sena, 2002).

A partir dessa percepção, Andrade et al. (2011) identificaram que a atuação direta de estudantes de medicina com a comunidade possibilita a valorização da pesquisa científica, visto que as atividades desenvolvidas junto à população são orientadas pela metodologia científica e, no segundo e terceiro anos, pela metodologia da problematização. Dessa forma, o estudante começa a leitura de textos científicos desde o início do curso, o que contribui para o seu embasamento teórico e a formulação de devolutivas que modifiquem a realidade da população-alvo (Andrade et al., 2011).

A inserção do estudante de medicina nos diferentes níveis de atenção à saúde possibilita o alcance da resolubilidade adequada à complexidade das atividades exercidas. O contato repetitivo do acadêmico com a atenção básica possibilita a capacitação para atuar como agente de transformação social, por meio de sua incorporação à produção de serviços daquele local (Andrade et al., 2011). 
É importante investir na transdisciplinaridade das disciplinas do curso com o objetivo de colocar, a serviço da sociedade, profissionais que visem aprimorar, além de aptidões teórico-técnicas, o desenvolvimento de habilidades sociais e pessoais, incentivando a avaliação e o exercício da autocrítica permanentemente no processo natural das atividades de todas as disciplinas básicas e profissionais (Siqueira et al., 2006 apud Carneiro et al., 2010).

\section{Considerações finais}

Este trabalho permitiu realizar a busca de literaturas atuais sobre a utilização de metodologias ativas de aprendizagem durante a graduação, a formação técnica ou na capacitação de profissionais da saúde. Perceberam-se, então, tecnologias inovadoras que contribuem significativamente para o processo de ensino-aprendizagem.

A aplicação dessas metodologias no ensino da graduação vem demonstrando benefícios na formação desses profissionais da saúde. Além disso, quando empregadas também na capacitação de trabalhadores, como na educação permanente, é capaz de transformar o contexto de trabalho na saúde.

Concluiu-se que a produção cientifica nesse âmbito é escassa, necessitando de um aprofundamento na literatura disponível. Por isso a importância de estudos abordando a temática, para identificar essas metodologias utilizadas, além de quantificar e qualificar as leituras sobre o assunto. Portanto, espera-se que o presente estudo instigue a realização de outras pesquisas na área.

\section{Colaboradores}

Os autores participaram de todas as etapas de produção do manuscrito. 
Resumen El presente artículo tiene como principal objetivo analizar la producción científica sobre el uso de metodologías activas de aprendizaje en la educación en la salud. Se trata de una revisión bibliográfica de la literatura, que utilizó para la selección de los artículos la base de datos de la Biblioteca Virtual en Salud. La investigación tuvo lugar en los meses de septiembre a noviembre de 2012. En la búsqueda se seleccionaron 15 artículos completos. Los resultados pusieron de manifiesto que el uso de las metodologías activas motiva al discente y lo orienta a buscar informaciones con el propósito de solucionar el impasse y fomentar su propio desarrollo, haciendo que éste observe que el nuevo aprendizaje es un instrumento necesario y significativo para ampliar sus posibilidades y caminos. Estas metodologías también han contribuido para cambios en el modo de pensar y actuar de los profesionales de la salud, los cuales pasaron a interactuar en un espacio plural de intereses, potencialidades y capacidades.

Palabras clave metodologías activas de aprendizaje; educación en salud.

\section{Notas}

1 Escola de Formação em Saúde da Família Visconde de Sabóia, Sobral, Ceará, Brasil. <cilenefreit@yahoo.com.br>

Correspondência: Rua Marechal Humberto de Alencar Castelo Branco, 447, Expectativa, CEP 62041-590, Sobral, Ceará, Brasil.

2 Universidade Estadual Vale do Acaraú, Programa de Educação pelo Trabalho para a Saúde, Programa Nacional de Reorientação da Formação Profissional em Saúde, Sobral, Ceará, Brasil.

<cibellyaliny@gmail.com>

3 Universidade Estadual Vale do Acaraú, Centro de Ciências da Educação, Sobral, Ceará, Brasil.

$<$ reginaldo.fp@hotmail.com>

4 Universidade Estadual Vale do Acaraú, Centro de Ciências da Saúde, Sobral, Ceará, Brasil.

$<$ miosawa@gmail.com>

5 Universidade Federal do Ceará, Programa de Pós-Graduação em Enfermagem, Fortaleza, Ceará, Brasil.

<gleicinha-lima@hotmail.com>

6 Universidade Federal do Ceará, Programa de Pós-Graduação em Saúde da Família, Sobral, Ceará, Brasil.

$<$ karininhamesquita@hotmail.com>

7 Universidade Estadual Vale do Acaraú, Centro de Ciências da Saúde, Sobral, Ceará, Brasil.

$<$ lanacoelho.svetlana@gmail.com>

8 Universidade Estadual Vale do Acaraú, Centro de Ciências da Saúde, Sobral, Ceará, Brasil.

<janicedavila@hotmail.com> 


\section{Referências}

ANDRADE, Silvia C. et al. Avaliação do desenvolvimento de atitudes humanísticas na graduação médica. Revista Brasileira de Educação Médica, Rio de Janeiro, v. 35, n. 4, dez. 2011.

ATHANAZIO, Rodrigo A. et al. Academética: um novo método de estudo continuado sobre ética médica e bioética. Revista Brasileira de Educação Médica, Rio de Janeiro, v. 28, n. 1, jan./abr. 2004.

BATISTA, Karina B. C.; GONÇALVES, Otilia S. J. Formação dos Profissionais de Saúde para o SUS. Saúde e Sociedade, São Paulo, v. 20, n. 4, p. 884-899, 2011.

BERBEL, Neusi A. N. A problematização e a aprendizagem baseada em problemas: diferentes termos ou diferentes caminhos?. Interface: Comunicação, Saúde, Educação, Botucatu, v. 2, n. 2, p. 139-54, 1998. Disponível em: <www.scielo.br/pdf/icse/v2n2/ 08.pdf>. Acesso em: 15 out. 2012.

BRASIL. Ministério da Saúde. Congresso. Senado. Resolução n. 4 CNE/CES, de 2001. Institui Diretrizes Curriculares Nacionais do Curso de Graduação em Medicina. Diário Oficial da União. Brasília, 9 nov. 2001. Seção 1, p. 38.

BRASIL. Ministério da Saúde. Portaria n. 198/GM/MS, de 13 de fevereiro de 2004. Institui a Política Nacional de Educação Permanente em Saúde como estratégia do SUS para a formação e o desenvolvimento de trabalhadores para o setor e dá outras providências. Brasília: Ministério da Saúde, 2004.

BRASIL. Ministério da Saúde. Secretaria de Gestão do Trabalho e da Educação na Saúde. Departamento de Gestão da Educação na Saúde. Política nacional de educação permanente em saúde. Brasília: Ministério da Saúde, 2009.

CARNEIRO, Larissa A. et al. O ensino da ética nos cursos de graduação da área da saúde. Revista Brasileira de Educação Médica, Rio de Janeiro, v. 34, n. 3, p. 412-421, jul./set.,
2010. Disponível em: <www.scielo.br/scielo. php?pid=S0100-55022010000300011\&script $=$ sci_arttext>. Acesso em: 18 out. 2012

CECCIM, Ricardo B. Educação permanente em saúde: desafio ambicioso e necessário. Interface: Comunicação, Saúde, Educação, São Paulo, v. 9, n. 16, p. 161-77, set. 2004.

CEZAR, Pedro H. N. et al. Transição paradigmática na educação médica. Um olhar construtivista dirigido à aprendizagem baseada em problemas. Revista Brasileira de Educação Médica, Rio de Janeiro, v. 32, n. 4, p. 298-303, 2010.

CHIESA, Anna M. et al. A formação de profissionais da saúde: Aprendizagem significativa à luz da promoção da saúde. Revista Cogitare Enfermagem, São Paulo, v. 12, n. 2, abr./jun. 2007.

FEUERWERKER, Laura C. M.; SENA, Roseni R. Contribuição ao movimento de mudança na formação profissional em saúde: uma avaliação das experiências UNI. Interface: Comunicação, Saúde, Educação, São Paulo, v. 6 , n. 10, p. 37-50, 2002.

FIGUEIRA, Eliandro J. G. et al. Apreensão dos tópicos de ética médica no ensino-aprendizagem de pequenos grupos. Comparando aprendizagem baseada em problemas com o modelo tradicional. Revista da Associação Médica Brasileira, São Paulo, v. 50, n. 2, p. 133-41, 2004.

FREIRE, Paulo. Pedagogia da autonomia: saberes necessários à prática educativa. 33 . ed. São Paulo: Paz e Terra, 2006.

FREIRE, Paulo. Pedagogia do oprimido. São Paulo: Paz e Terra, 1996.

FREITAS, Valeria P. et al. Mudança no processo ensino aprendizagem nos cursos de graduação em odontologia com utilização de metodologias ativas de ensino e aprendizagem. RFO UPF, Passo Fundo, v. 14, n. 2, p. 163-167, maio/ago. 2009. 
FUSCALDI-CORRÊA, Thiago et al. Novas metodologias de integração do ensino de saúde pública na faculdade de medicina utilizando a informática. Revista Médica de Minas Gerais, Belo Horizonte, v. 18, supl. 4, p. 67-73, 2008.

GOMES, Annatalia M. A.; MOURA, Escolástica R. F. M.; AMORIM, Rosendo F. O lugar da ética e bioética nos currículos de formação médica. Revista Brasileira de Educação Médica, Rio de Janeiro, v. 30, n. 2, p. 56-65, 2006. Disponível em: <www. scielo.br/scielo.php?script=sci_arttext\&pid= S0100022006000200008\&lng =pt\&nrm $=$ iso \& tlng =pt $>$. Acesso em: 25 set. 2012.

GOMES, Maria P. C. et al. O uso de metodologias ativas no ensino de graduação nas ciências sociais e da saúde: avaliação dos estudantes. Ciência e Educação, Bauru, v. 16, n. 1, p. 181-198, 2010.

HAGUENAUER, Cristina. Metodologias e estratégias na educação a distância. Latec. Adaptado da entrevista concedida à Folha Dirigida, em janeiro de 2005. Disponível em: $<$ www.latec.ufrj.br/portfolio/at/4\%20EAD $\% 20$ metodologias $\mathrm{pdf}>$. Acesso em: 20 set. 2012.

MACHADO, Maria H. Mercado de trabalho em saúde. In: FALCÃO, André et al. (Org.). Observatório de recursos humanos em saúde no Brasil: estudos e análises. Rio de Janeiro: Editora Fiocruz, 2003. p. 227-248.

MARCONI, Marina A.; LAKATOS, Eva M. Metodologia científica. 4. ed. São Paulo: Atlas, 2004.

MARIN, Maria J. S. et al. Pós-graduação multiprofissional em saúde: resultados de experiências utilizando metodologias ativas. Interface: Comunicação, Saúde, Educação, Botucatu, v. 14, n. 33, p. 331-44, abr./jun. 2010.

MITRE, Sandra M. et al. Metodologias ativas de ensino-aprendizagem na formação profissional em saúde: debates atuais. Ciência \& Saúde Coletiva, Rio de Janeiro, v. 13, supl. 2, p. 2.133-2.144, 2008.
MUNGUBA, Marilene C. S. Educação na saúde: sobreposição de saberes ou interface?. Revista Brasileira em Promoção da Saúde, Fortaleza, v. 23, n. 4, p. 295-296, out./dez. 2010.

OLIVEIRA, Fernanda M. C. S. N. et al. Educação permanente e qualidade da assistência à saúde: aprendizagem significativa no trabalho da enfermagem. Aquichan, Bogotá, v. 11, n. 1, abr. 2011.

PEDROSA, Ivanilda L. et al. Uso de metodologias ativas na formação técnica do agente comunitário de saúde. Trabalho, Educação e Saúde, Rio de Janeiro, v. 9, n. 2, p. 319-332, jul./out. 2011.

RIBEIRO, Victoria M. B. Discutindo o conceito de inovação curricular na formação dos profissionais de saúde: o longo caminho para as transformações do ensino médico. Trabalho, Educação e Saúde, Rio de Janeiro, v. 3, n. 1, p. 91-121, 2005.

RODRIGUES, Livia P. F.; RONCADA, Maria J. Educação nutricional no Brasil: evolução e descrição de proposta metodológica para escolas. Comunicação em Ciências Saúde, Brasília, v. 19, n. 4, p. 315-322, 2008.

SAKAI, Marcia H.; LIMA, Gerson Z. PBL: Uma visão geral do método. Olho mágico, Londrina, v. 2, n. 5/6 (Número especial), 1996.

SIQUEIRA-BATISTA, Rodrigo; SIQUEIRA-BATISTA, Rômulo. Os anéis da serpente: a aprendizagem baseada em problemas e as sociedades de controle. Ciência \& Saúde Coletiva, Rio de Janeiro, v. 14, n. 4, p. 1.183-1.192, 2009.

SIQUEIRA, José E. O ensino da bioética no curso médico. Bioética, v. 11, n. 2, p. 33-42, 2003.

Recebido em 16/06/2014

Aprovado em 30/03/2015 